\title{
Chemotherapy Center
}

National Cancer Institute

\section{Source}

National Cancer Institute. Chemotherapy Center. NCI Thesaurus. Code C51967.

A health care organization staffed, equipped, and licensed for performing chemotherapy, biological therapy and associated oncology care. 